\title{
Improving the Ranking Performance of Decision Trees
}

\author{
Bin Wang and Harry Zhang \\ Faculty of Computer Science, University of New Brunswick, Canada \\ \{bin.wang, hzhang\}@unb.ca
}

\begin{abstract}
An accurate ranking of instances based on their class probabilities, which is measured by AUC (area under the Receiver Operating Characteristics curve), is desired in many applications. In a traditional decision tree, two obstacles prevent it from yielding accurate rankings: one is that the sample size on a leaf is small, and the other is that the instances falling into the same leaf are assigned to the same class probability. In this paper, we propose two techniques to address these two issues. First, we use the statistical technique shrinkage which estimates the class probability of a test instance by using a linear interpolation of the local class probabilities on each node along the path from leaf to root. An efficient algorithm is also brought forward to learn the interpolating weights. Second, we introduce an instance-based method, the weighted probability estimation (WPE), to generate distinct local probability estimates for the test instances falling into the same leaf. The key idea is to assign different weights to training instances based on their similarities to the test instance in probability estimation. Furthermore, we combine shrinkage and WPE together to compensate for the defects of each. Our experiments show that both shrinkage and WPE improve the ranking performance of decision trees, and that their combination works even better. The experiments also indicate that various decision tree algorithms with the combination of shrinkage and WPE significantly outperform the original ones and other state-of-the-art techniques proposed to enhance the ranking performance of decision trees.
\end{abstract}

Keywords: Decision Tree, Class Probability, Ranking, AUC, Shrinkage, $W P E$

\section{Introduction}

Decision trees have been regarded as one of the most popular models in the fields of machine learning and data mining. Traditionally, accuracy is often used to evaluate the classification performance of decision trees. However, it is not sufficient to merely classify an instance into the most possible class in many applications. A ranking of instances based on the class probability $P(c \mid e)$, the probability of an instance $e$ in the class $c$, is more desirable. For example, a credit card company can consider the top $\mathrm{X} \%$ in a ranking of applicants, who are most likely to belong to the profitable class. In this paper, we use AUC (Area 
Under the Receiver Operating Characteristics Curve) to evaluate the ranking performance of decision trees, which has received considerable attention as a measure of ranking $14|8| 6]$.

Accurate probability estimation certainly leads to accurate ranking which is based on the class probabilities. Unfortunately, decision trees, such as C4.5 [15], have been observed to produce poor probability estimates which result in the poor ranking performance [11313. In a decision tree, the class probability $P(c \mid e)$ is estimated by the fraction of instances of class $c$ on the leaf which $e$ falls into. It causes two problems [17]. One is the high bias: decision tree growing methods try to make leaves pure, so the probability estimates on leaves are shifted towards zero or one; the other is the high variance: the training instances on a leaf are often not enough to provide reliable probability estimates. Besides, decision tree algorithms often assign the same class probability to the instances falling into the same leaf. The ranks of the instances with equal probability are generated randomly, and thus the AUC score tends to decrease.

In this paper, we introduce shrinkage and the weighted probability estimation $(W P E)$ to solve the above problems. The probability estimate with shrinkage for a test instance is decided by the linear interpolation of the local probability estimates on each node along the path from leaf to root, instead of merely being decided by the leaf. An efficient algorithm is proposed to determine the interpolating weights. WPE is an instance-based method, which assigns the distinct class probabilities to the instances falling into the same node, and thus leads to better ranking performance. However, there are still some flaws in shrinkage and $W P E$. We combine these two techniques together to compensate for the defects of each. Shrinkage, WPE and their combination are applicable to any decision tree algorithms without changing the tree-building process and tree structure. We design empirical experiments to verify that both shrinkage and WPE can improve the ranking performance of traditional decision tree algorithms, such as C4.5 15] and C4.4 12, in terms of AUC. We also show that the combination of these two techniques is even stronger than either single one, and according to AUC outperforms other techniques such as $m$-Branch [5], bagging [12] and Ling's algorithm 9], which aim to improve the probability-based ranking in decision trees.

The rest of the paper is organized as follows. In Section 2, we discuss the related work on improving the probability-based ranking performance of decision trees. In Section 3, we describe shrinkage and the algorithm for training the interpolating weights. In Section 4, we illuminate the process of WPE. In Section 5, we show how to combine shrinkage and WPE together. In Section 6, the experiments and results are presented and analyzed. Finally, we summarize our work and bring forward the future research in Section 7.

\section{Related Work}

As the foregoing analysis has shown, the ranking performance of $\mathrm{C} 4.5$ is poor (i.e., low AUC score). Provost and Domingos [12] utilize two techniques to 
improve the AUC of C4.5. The first is to turn off pruning and the second is to use Laplace correction. They call the resulting algorithm C4.4. However, turning off pruning results in a large tree so that the number of training instances on each leaf tends to be small. Then the corresponding probability estimation could be unreliable even when using Laplace correction. Moreover, the same probability estimate is assigned on the same leaf in C4.4. Bagging is also used to improve the AUC of decision trees [213, but the results produced by bagging are not comprehensible.

Some researchers have noticed that the information used for estimating the probability of an instance should not be limited to the leaf which the instance falls into. Ling and Yan 9] present an algorithm to average probability estimates from all leaves, instead of a single leaf. The contribution of each leaf is determined by the deviation in attribute values from root to leaf. But the deviation they described has only been reflected by a "confusion factor" which can be regarded as the probability of errors that alters the attribute values. Although it produces distinct probability estimates for the instances on the same leaf, setting up good "confusion factors" could still be an issue. Moreover, the algorithm should go through the whole tree to calculate the contribution of each leaf. Consequently, the complexity of this algorithm tends to be fairly high.

Ferri et al. [5] introduce the $m$-Branch method to smooth the probability estimates on leaves with the history information along the paths. $M$-Branch is a recursive process in which the probability estimate on the parent node is put into the probability estimate on the child node. The parameters of $m$ Branch are adjusted by the height of a node and the cardinality (the number of instances associated with a node). Although they notice that the information from other nodes should be utilized, they still assign the same class probability to the instances on the same leaf.

Zadrozny and Elkan [17] suggest a method called curtailment, to improve the probability estimation of decision trees. In curtailment, if a leaf contains few training instances and can not induce reliable probability estimates, probability estimation can be raised to an ancestor node of this leaf, in which there are enough training instances. Curtailment blurs the distinction between internal nodes and leaves, because a node may serve as an internal node which owns child nodes, or serve as a leaf which assigns the same probability estimate to instances. Curtailment is reminiscent of the methods proposed by Bahl et al. [1] and Buntine 4] that calculate a weighted average of training frequencies at nodes along the path from root to leaf. However, they do not propose an effective algorithm to learn the weights. Hastie and Pregibon [7] provide a shrinking process called recursive shrinking to smooth the pruning in decision trees. The shrinking process is parameterized by a scalar $\theta$ which must be specified based on the data.

Shrinkage has been brought into text classification [10, in which the word probability estimates are improved by shrinkage in a hierarchical structure. In this method, the final class probabilities are estimated by naive Bayes. Besides, the weights of shrinkage are determined by an EM algorithm. The EM algorithm 
needs an iterative procedure that converges usually after many iterations. Thus, it is quite time-consuming.

\section{Shrinkage}

Figure 1 shows a sample decision tree, which has five internal nodes $N_{1}, \ldots, N_{5}$ and six leaves $N_{6}, \ldots, N_{11}$, associated with the subsets of training instances $D_{1}$, $\ldots, D_{11}$.

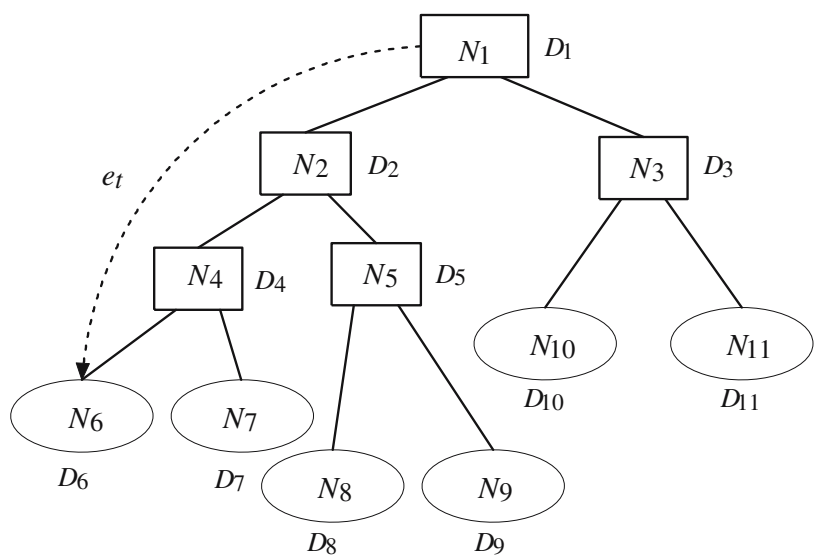

Fig. 1. A sample of decision tree

Assume that the test instance $e_{t}$ falls into leaf $N_{6}$ passing internal nodes $N_{1}, N_{2}$ and $N_{4}$. In the traditional decision tree algorithm, the class probability $P\left(c_{j} \mid e_{t}\right)$ is estimated by the fraction of training instances in class $c_{j}$ on leaf $N_{6}$. The shrinkage estimate of $P\left(c_{j} \mid e_{t}\right)$ is a linear combination of the local class probability estimates on the nodes $N_{1}, N_{2}, N_{4}$ and $N_{6}$. Given class $c_{j}$, the local class probability of $e_{t}$ on the $i t h$ node is estimated as follows.

$$
P^{i}\left(c_{j} \mid e_{t}\right)=\frac{n_{j}+1 /|C|}{\left|D_{i}\right|+1},
$$

where $n_{j}$ is the number of training instances belonging to class $c_{j},|C|$ is the number of classes, and $\left|D_{i}\right|$ is the number of training instances on the $i t h$ node. In Equation 11. Laplace correction is used to smooth the probability estimate towards the uniform distribution of class labels.

Although the root $N_{1}$ contains all the training instances, it probably has few instances whose class labels are rare, which may result in unreliable probability estimation. Therefore, we extend the tree by adding a uniform node $N_{0}$ beyond the root [10, on which the uniform distribution of instances is adopted. In order to keep the consistency of expression, we define the class probability of $e_{t}$ given class $c_{j}$ on the uniform node $N_{0}$ as $P^{0}\left(c_{j} \mid e_{t}\right)=\frac{1}{\left|D_{1}\right|}$, where $\left|D_{1}\right|$ is the number 
of training instance on root $N_{1}$. The shrinkage estimate of $P\left(c_{j} \mid e_{t}\right)$ is shown as follows.

$P\left(c_{j} \mid e_{t}\right)=w_{j}^{0} P^{0}\left(c_{j} \mid e_{t}\right)+w_{j}^{1} P^{1}\left(c_{j} \mid e_{t}\right)+w_{j}^{2} P^{2}\left(c_{j} \mid e_{t}\right)+w_{j}^{3} P^{3}\left(c_{j} \mid e_{t}\right)+w_{j}^{4} P^{4}\left(c_{j} \mid e_{t}\right)$,

where $P^{1}\left(c_{j} \mid e_{t}\right), P^{2}\left(c_{j} \mid e_{t}\right), P^{3}\left(c_{j} \mid e_{t}\right), P^{4}\left(c_{j} \mid e_{t}\right)$ are the local probability estimates on $N_{1}, N_{2}, N_{4}, N_{6}$ respectively, $w_{j}^{0}, w_{j}^{1}, w_{j}^{2}, w_{j}^{3}, w_{j}^{4}$ are the interpolating weights for class $c_{j}$ assigned to the corresponding nodes, in which $\sum_{m=0}^{4} w_{j}^{m}=1$. Figure 2 shows the path, probabilities and related weights. Equation 2 can be extended to deal with the general case which has $k$ nodes on the path.

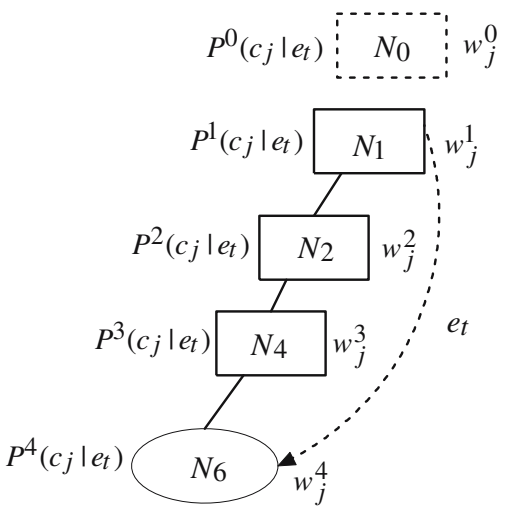

Fig. 2. The path of $e_{t}$ in the decision tree. Uniform node is added above $N_{1}$. The local probabilities are estimated on each node, and the interpolating weights are assigned to each node.

Shrinkage represents a tradeoff between the specificity and generality. At a leaf, since the probability estimates are yielded based on the training instances that come through a series of partitions on the internal nodes, they are more specific but less general than the ones on the ancestors of the leaf. At the root, the estimates are more general because all the training instances are included, but they are less specific than the estimates on the descendants of the root.

A key problem to apply shrinkage is to determine the interpolating weights effectively and efficiently. Assume that $N_{0}, N_{1}, \ldots, N_{k}$ is a path, where $N_{0}$ is the uniform node, $N_{1}$ is the root, and $N_{k}$ is the leaf. Given class label $c_{j}$, let $\beta_{j}^{0}$, $\beta_{j}^{1}, \ldots, \beta_{j}^{k}$ be the influence degree of node $N_{i}$ to class $c_{j}$, and $w_{j}^{0}, w_{j}^{1}, \ldots, w_{j}^{k}$ be the interpolating weights. We use the following algorithm to determine the interpolating weights.

Algorithm DIW (path, D, $c_{j}$ )

Input: path is a sequence of nodes $N_{0}, N_{1}, \ldots, N_{k}, D$ is the training set, and $c_{j}$ is a class label.

Ouput: A set of interpolating weights for $c_{j}$ for all nodes $N_{0}, N_{1}, \ldots, N_{k}$. 
Step 1: Initialize each weight $w_{j}^{i}$ as $\frac{1}{k+1}$ so that $\sum w_{j}^{i}=1$, and initialize each degree $\beta_{j}^{i}$ to zero. Set each training instance on leaf unmarked.

Step 2: Choose an unmarked training instance $x$ on leaf $N_{k}$, remove $x$ from all the training subsets $D_{1}, \ldots, D_{k}$ on each node along the path.

Step 3: Set $P^{0}\left(c_{j} \mid x\right)=1 /\left|D_{1}\right|$, where $\left|D_{1}\right|$ is the number of training instances on root $N_{1}$. From $N_{1}$ to $N_{k}$, estimate the local class probabilities $P^{i}\left(c_{j} \mid x\right)(i=$ $1, \ldots, k)$ using Equation 1 .

Step 4: For each node, update its degree as follows:

$$
\beta_{j}^{i}=\beta_{j}^{i}+\frac{w_{j}^{i} P^{i}\left(c_{j} \mid x\right)}{\sum_{m=0}^{k} w_{j}^{m} P^{m}\left(c_{j} \mid x\right)}, i=0, \ldots, k .
$$

Step 5: Mark instance $x$ and put $x$ back to each training subset. If there is an un-marked training instance on $N_{k}$, go back to Step 2.

Step 6: Compute $w_{j}^{i}$ by normalizing the set of degrees $\left\{\beta_{j}^{0}, \beta_{j}^{1}, \ldots, \beta_{j}^{k}\right\}$ as follows:

$$
w_{j}^{i}=\frac{\beta_{j}^{i}}{\sum_{m=0}^{k} \beta_{j}^{m}}, i=0, \ldots, k .
$$

Return: $\left\{w_{j}^{0}, w_{j}^{1}, \ldots, w_{j}^{k}\right\}$.

Note that on a node $N_{i}$, the local class probabilities $P^{i}\left(c_{j} \mid x\right)\left(x \in D_{k}\right)$ have the same estimate in Step 3, since the fraction of training instance in class $c_{j}$ on $N_{i}$ is used in Equation 11. Because of this, Algorithm DIW is not able to be adapted to a multiple-iteration algorithm. The interpolating weights are returned only after one iteration (go through each instance on the leaf once).

When we apply shrinkage to a decision tree algorithm, a decision tree is built by that algorithm first. Then the Algorithm DIW is applied to each path to set up the interpolating weights for each node and each class label. Given a test instance $e_{t}$, it is sorted down to a leaf, and then its class probability $P\left(c_{j} \mid e_{t}\right)$ is computed using Equation 2.

\section{Weighted Probability Estimation}

As mentioned before, a major issue for decision trees is that all the instances falling into the same leaf will have the same probability estimate, which is an obstacle to yielding accurate ranking. We notice that the instance-based method can generate the distinct and local estimates.

We introduce WPE, an instance-based method, to estimate the class probabilities on the leaves. Given a test instance $e_{t}$ which consists of a set of attribute values and a class label, $e_{t}$ falls into a leaf $L$. We define the similarity between $e_{t}$ and a training instance $e_{r}$ on $L$ as follows.

$$
\operatorname{sim}\left(e_{t}, e_{r}\right)=\sum_{i=1}^{n} e q u\left(A_{i}\left(e_{t}\right), A_{i}\left(e_{r}\right)\right),
$$


where $n$ is the number of attributes, $e q u(a, b)$ is a boolean function whose value is either $1(a=b)$ or $0(a \neq b)$, and $A_{i}(e)$ is the $i t h$ attribute value of $e$. In $W P E$, we calculate $\operatorname{sim}\left(e_{t}, e_{r}\right)$ for each training instance $e_{r}$ on $L$ as the weight of $e_{r}$ and estimate $P\left(c_{j} \mid e_{t}\right)$ as follows.

$$
P\left(c_{j} \mid e_{t}\right)=\frac{\sum_{e_{r} \in D_{L}, C\left(e_{r}\right)=c_{j}}\left(\operatorname{sim}\left(e_{t}, e_{r}\right)+1\right)+1 /|C|}{\sum_{e_{r} \in D_{L}}\left(\operatorname{sim}\left(e_{t}, e_{r}\right)+1\right)+1},
$$

where $D_{L}$ is the training instance subset on $L, C\left(e_{r}\right)$ is the class label of $e_{r}$. At the numerator of Equation 6, the total weights of the instances which belong to class $c_{j}$ in $D_{L}$ is computed, and the denominator is roughly the total weights of all the instances in $D_{L}$. Equation 6 uses the same Laplace correction as Equation 1.

Without changing the process of building a decision tree, we return the class probabilities from the leaves, which are estimated by WPE. Note that, for different test instances $e_{t 1}$ and $e_{t 2}$ falling into the same leaf, $P\left(c_{j} \mid e_{t 1}\right)$ and $P\left(c_{j} \mid e_{t 2}\right)$ could be different based on Equation 6. This means that we could make distinct probability estimates for the instances on the same node, which is the key to obtaining accurate ranking of instances.

\section{Combination of Shrinkage and Weighted Probability Estimation}

Both shrinkage and WPE are able to make up for the deficiencies of decision tree algorithms. Shrinkage breaks the restriction that the probabilities only can be estimated on the leaves, and WPE solves the problem that the different test instances falling into the same leaf are assigned to the same class probability estimate. However, they still suffer from some problems. In Algorithm DIW, the same probability estimate is assigned to different instances on the same node in Step 3. The generated interpolating weights for shrinkage are not effective enough. Moreover, when the sizes of training subsets on leaves are small, $W P E$ could not generate reliable class probability estimates without the support of other nodes on the paths. The combination of shrinkage and WPE could compensate for the weakness of each. We illuminate the whole process of decision tree algorithm with the combination as follows.

\section{Training:}

First, a decision tree is built by a traditional decision tree algorithm. Then, Algorithm DIW is carried out to train the interpolating weights for shrinkage. We use WPE to estimate the class probabilities $P^{i}\left(c_{j} \mid x\right)$ for a training instance $x$ on the leaf in Step 3 of Algorithm DIW. Here $x$ is treated as a test instance, and $P^{i}\left(c_{j} \mid x\right)$ is estimated by Equation [6 (instead of Equation 11). When the similarity is calculated using Equation 5. we compare all of the attribute values and the class labels, since the class label is known for $x$.

\section{Testing:}

Given a test instance $e_{t}$, it is sorted along a path from the root to a leaf. The local class probabilities $P^{i}\left(c_{j} \mid e_{t}\right)$ are estimated by WPE (Equation 6). We do not use 
the class label to calculate the similarity in Equation 5, since the class label is unknown for $e_{t}$. Finally, the returned class probability $P\left(c_{j} \mid e_{t}\right)$ is estimated by shrinkage (Equation 2) with the interpolating weights determined by Algorithm DIW and the local probabilities estimated by WPE.

Using WPE in Step 3 of Algorithm DIW, the distinct probability estimates are assigned to the instances on the same node so that the returned interpolating weights are more effective. This also makes it possible that Algorithm DIW can be adapted to a multiple-iteration algorithm, which terminates when the interpolating weights are converged. The multiple-iteration algorithm appears like an EM algorithm. However, a typical EM algorithm takes thousands of iterations to converge, which is fairly time-consuming. In the experiments, we still use the single-iteration Algorithm DIW. The experimental results show that the outcome of weights after many iterations is not significantly better than the one from just one iteration.

\section{Experiments and Results}

Our experiments are conducted on 35 data sets from Weka [16, which come from the UCI repository. We download these data sets in the format of ar $f f$ from the website of Weka. There are some preprocessing stages adopted on each data set. First, we use the filter ReplaceMissingValues in Weka to replace the missing values of attributes in each data set. Second, we use the filter Discretize, the unsupervised ten-bin discretization in Weka, to discretize numeric attributes. Thus, all the attributes are treated as nominal. Third, we notice that, if the number of values of an attribute is almost equal to the number of instances in a data set, this attribute does not contribute any information to the purpose of prediction. So we use the filter Remove in Weka to delete this type of attribute.

In the first experiment, we compare the algorithms, such as $\mathrm{C} 4.5$ with combination of shrinkage and WPE (C45-C), C4.5 with shrinkage (C45-S), C4.5 with WPE (C45-W), C4.5 (C45), C4.5 with m-Branch (C45-M), C4.5 with LingYan's algorithm (C45-L), C4.5 with bagging (C45-B). In the second experiment, we compare the algorithms, such as $\mathrm{C} 4.4$ with the combination of shrinkage and WPE (C44-C), C4.4 with shrinkage (C44-S), C4.4 with WPE (C44-W), C4.4 (C44), C4.4 with $m$-Branch (C44-M), C4.4 with LingYan's algorithm (C44-L), C4.4 with bagging (C44-B). We implement shrinkage, WPE, the combination 1 , C4.4, m-Branch, LingYan's algorithm and AUC evaluation within the Weka framework, and use the implementation of C4.5 and bagging in Weka. In all experiments, the AUC score of an algorithm on a data set is obtained via 5 runs of ten-fold cross validation. Runs with the various algorithms are carried out on the same training data sets and evaluated on the same test data sets. Finally, we conduct two-tailed $t$-test with a $95 \%$ confidence level to compare each pair of algorithms.

${ }^{1}$ Codes for shrinkag, WPE and their combination are available at http://www.cs. unb.ca/ hzhang/ShrinkageCode.rar 
Table 3 and Table 4 show the AUC scores of the algorithms on each data set. The two-tailed $t$-test results are shown in Table 1 and Table 2 Each entry of Table 1 and Table 2 has the format of $w / t / l$. It means that compared with the algorithm in the corresponding column, the algorithm in the corresponding row wins in $w$ data sets, ties in $t$ data sets and loses in $l$ data sets. From our experiments, we have the following observations:

- C45-S and C44-S outperform C45 and C44 respectively. C45-W and C44-W outperform $\mathrm{C} 45$ and $\mathrm{C} 44$ respectively.

- Either shrinkage or WPE is not perfect compared to some techniques. C45$\mathrm{S}$ is worse than $\mathrm{C} 4.5$ with other techniques. $\mathrm{C} 44-\mathrm{S}$ is not as good as $\mathrm{C} 44-\mathrm{M}$ and $\mathrm{C} 44-\mathrm{B}$. C44-W is worse than $\mathrm{C} 4.4$ with other techniques.

- C45-C outperforms C45 and C4.5 with any other techniques. C44-C outperforms $\mathrm{C} 44$ and $\mathrm{C} 4.4$ with any other techniques.

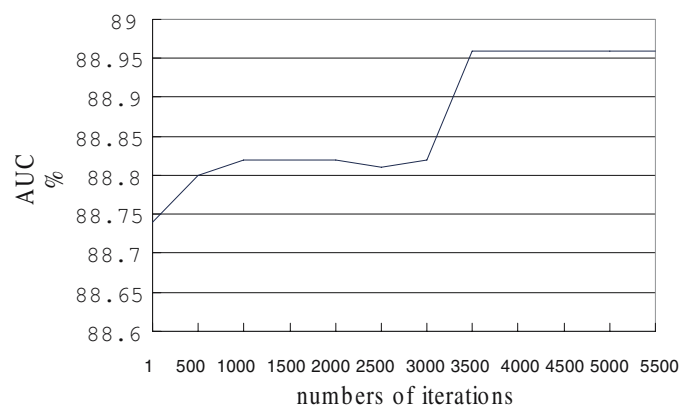

Fig. 3. The AUC scores corresponding to the different numbers of iterations

The experimental results are not surprising. Due to assigning the same local probability estimates on each node, Algorithm DIW could not return more effective interpolating weights, so shrinkage is worse than other techniques. Although WPE is able to estimate the probability distinctly, it suffers from the case when the sizes of training subsets on leaves are small, and that's why $\mathrm{C} 44-\mathrm{W}$ is worse than $\mathrm{C} 4.4$ with other techniques. The combination of shrinkage and WPE solves the above problems: First, applying WPE in Algorithm DIW

Table 1. Summary of comparisons for the algorithms related with C4.5

\begin{tabular}{l|l|l|l|l|l|l}
\hline & $\mathrm{C} 45-\mathrm{C}$ & $\mathrm{C} 45-\mathrm{S}$ & $\mathrm{C} 45-\mathrm{W}$ & $\mathrm{C} 45$ & $\mathrm{C} 45-\mathrm{M}$ & $\mathrm{C} 45-\mathrm{L}$ \\
\hline $\mathrm{C} 45-\mathrm{S}$ & $0 / 5 / 30$ & & & & & \\
$\mathrm{C} 45-\mathrm{W}$ & $3 / 18 / 14$ & $19 / 16 / 0$ & & & & \\
$\mathrm{C} 45$ & $0 / 5 / 30$ & $3 / 18 / 14$ & $0 / 4 / 31$ & & & \\
$\mathrm{C} 45-\mathrm{M}$ & $2 / 9 / 24$ & $8 / 27 / 0$ & $1 / 17 / 17$ & $16 / 19 / 0$ & & \\
$\mathrm{C} 45-\mathrm{L}$ & $0 / 10 / 25$ & $14 / 15 / 6$ & $2 / 17 / 16$ & $19 / 13 / 3$ & $10 / 18 / 7$ & \\
$\mathrm{C} 45-\mathrm{B}$ & $2 / 7 / 26$ & $14 / 18 / 3$ & $4 / 17 / 14$ & $24 / 11 / 0$ & $10 / 22 / 3$ & $7 / 20 / 8$ \\
\hline
\end{tabular}


Table 2. Summary of comparisons for the algorithms related with C4.4

\begin{tabular}{l|l|l|l|l|l|l}
\hline & C44-C & C44-S & C44-W & C44 & C44-M & C44-L \\
\hline C44-S & $2 / 14 / 19$ & & & & & \\
C44-W & $0 / 12 / 23$ & $3 / 24 / 8$ & & & & \\
C44 & $1 / 11 / 23$ & $0 / 11 / 24$ & $4 / 20 / 11$ & & & \\
C44-M & $2 / 14 / 19$ & $7 / 24 / 4$ & $7 / 27 / 1$ & $16 / 19 / 0$ & & \\
C44-L & $0 / 14 / 21$ & $4 / 24 / 7$ & $10 / 16 / 9$ & $14 / 16 / 5$ & $7 / 20 / 8$ & \\
C44-B & $2 / 17 / 16$ & $9 / 24 / 2$ & $7 / 27 / 1$ & $19 / 16 / 0$ & $6 / 29 / 0$ & $9 / 25 / 1$ \\
\hline
\end{tabular}

Table 3. Experimental results on AUC for the algorithms related with C4.5

\begin{tabular}{|c|c|c|c|c|c|c|c|}
\hline Data set & C45-C & C45-S & C45-W & $\mathrm{C} 45$ & C45-M & C45-L & C45-B \\
\hline anneal & 95.91 & 90.45 & 95.24 & 83.45 & 89.73 & 93.70 & 86.49 \\
\hline 1.0 & .61 & .42 & 18 & 5.00 & .04 & 1.33 & 6.53 \\
\hline$g y$ & 66 & .81 & 0.08 & 1.54 & 2.68 & 9.98 & 4.86 \\
\hline & .07 & 3.11 & 5.22 & 73.84 & 3.95 & 9.93 & 77.91 \\
\hline ce-scale & .00 & 5.01 & 63.34 & 52.72 & 4.94 & 67.76 & 59.20 \\
\hline & 8 & .89 & 2.21 & .86 & 2.73 & 7.54 & 4.97 \\
\hline & 45 & .62 & 8.64 & .43 & 7.36 & 3.24 & 8.12 \\
\hline & .01 & .42 & 1.35 & 1.17 & 5.41 & 5.63 & 85.32 \\
\hline G & .42 & .50 & 0.25 & 3.56 & 5.38 & 0.96 & 87.68 \\
\hline & .46 & .71 & 4.54 & 3.17 & 9.82 & 1.26 & 1.31 \\
\hline & .49 & .90 & .35 & .48 & 2.32 & .08 & 4.16 \\
\hline & 68 & .69 & .47 & 5.26 & .05 & 9.33 & 9.74 \\
\hline & 61 & .93 & .60 & 7.11 & 88 & 2.46 & 1.26 \\
\hline & .25 & 3.27 & 3.89 & 3.16 & 3.31 & 3.85 & 3.75 \\
\hline & .58 & .01 & .40 & 0.91 & .02 & 3.76 & 3.77 \\
\hline & 98 & .65 & .01 & .65 & .48 & 17 & 6.39 \\
\hline & 42 & .61 & .78 & .43 & .50 & 2.90 & 1.54 \\
\hline yroid & 12 & .50 & .33 & 3.56 & 3.74 & 82.05 & 3.31 \\
\hline ere & & .29 & 25 & & .57 & .32 & 4.72 \\
\hline & 68 & 12 & 41 & 99 & .67 & 12 & 9.00 \\
\hline & .73 & .56 & .93 & .81 & .88 & 3.90 & 9.91 \\
\hline & 50 & .75 & .50 & 3.25 & 2.75 & 5.42 & 4.46 \\
\hline & & .47 & .90 & 1.16 & 6.29 & 7.33 & 3.02 \\
\hline & 0.00 & 100.00 & 00.00 & 0.00 & 00.00 & 9.46 & 00.00 \\
\hline & & 73 & 72.67 & & 67 & 29 & 9.01 \\
\hline & 60 & 81 & 99.24 & .47 & 8.97 & 6.19 & 99.33 \\
\hline & 12 & .69 & 96.67 & 3.42 & 4.48 & 94.90 & 94.01 \\
\hline & 84 & .05 & 76.26 & .49 & 4.14 & 75.33 & 81.92 \\
\hline & & & & & 96 & & .99 \\
\hline & & & & & 21 & 90 & 8.52 \\
\hline & .74 & .59 & .66 & 3.13 & 7.57 & 1.49 & 9.03 \\
\hline & 59 & .20 & .60 & .57 & 7.53 & 98.23 & 97.64 \\
\hline & & 00 & 95.32 & 2.83 & 5.61 & 2.96 & 96.40 \\
\hline wave & & 79 & 00 & 4.63 & .54 & .92 & 91.03 \\
\hline zoo & 88.48 & 80.38 & 88.00 & 79.57 & 80.29 & 85.76 & 80.62 \\
\hline
\end{tabular}

can generate the different probability estimates on each node for each training instances on the leaf; Second, shrinkage balances the probability estimation towards the nodes with large training subsets; Third, the class probability for a test instance is estimated by the local probabilities from WPE and shrinkage weights from Algorithm DIW.

In another interesting experiment, we adapt Algorithm DIW in C45-C to a multiple-iteration algorithm. For data set "Vehicle", the AUC scores from the different numbers of iterations are plotted in Figure 3. We observe that the AUC score is not changed at $88.96 \%$ after 3500 iterations. Compared with the AUC score $88.74 \%$ from a single iteration, we can see that the result after many iterations is not significantly better than the one from a single iteration. 
Table 4. Experimental results on AUC for the algorithms related with C4.4

\begin{tabular}{|c|c|c|c|c|c|c|c|}
\hline Data set & C44-C & C44-S & C44-W & $\mathrm{C} 44$ & C44-M & C44-L & C44-B \\
\hline anneal & 96.02 & 94.71 & 94.77 & 93.95 & 94.13 & 93.67 & 94.83 \\
\hline $1 . \mathrm{OH}$ & .61 & .54 & 3.43 & 2.11 & 93.56 & 92.25 & 93.25 \\
\hline ogy & .28 & .42 & 0.08 & 5.91 & 7.00 & 0.45 & 9.69 \\
\hline & .12 & 3.68 & 4.92 & 1.28 & 4.31 & 0.43 & 4.91 \\
\hline ce-scale & .50 & 7.76 & 6.79 & 9.42 & 57.90 & 66.45 & 5.15 \\
\hline & & 1 & 2.60 & .44 & .43 & 7.78 & 4.09 \\
\hline & & 58 & .59 & 3.08 & .18 & 3.27 & 8.79 \\
\hline & & .15 & 5.29 & 4.13 & 87.22 & 5.69 & 38.06 \\
\hline DRIG & & .80 & 3.45 & 2.43 & 3.60 & 0.66 & 6.00 \\
\hline & & .99 & 9.43 & 8.30 & 1.31 & 1.26 & 0.42 \\
\hline & & 96 & .67 & 06 & 2.70 & 5.04 & 3.44 \\
\hline & & 91 & .74 & 66 & .65 & 9.98 & 8.78 \\
\hline & & 11 & .29 & 0.57 & .23 & 2.06 & 9.52 \\
\hline & & .29 & 3.35 & 3.19 & 3.47 & 3.84 & 3.65 \\
\hline & & 52 & 3.45 & 3.21 & 3.67 & 3.82 & 3.65 \\
\hline & & 24 & .33 & 2.82 & 59 & 3.66 & 6.73 \\
\hline & 62 & 29 & .62 & 8.64 & 76 & 7.92 & 3.03 \\
\hline & & .31 & 13 & 2.23 & 3.74 & 1.40 & 2.28 \\
\hline & & & & .17 & .46 & .28 & 5.17 \\
\hline & & 0 & 93 & 3.52 & 85 & 7.33 & 8.68 \\
\hline & & 5 & 96 & 9.95 & 9.91 & 8.75 & 9.97 \\
\hline & 2 & .96 & .58 & 4.63 & 6.29 & 4.04 & 9.42 \\
\hline & 78 & .12 & 87.04 & 5.44 & 7.22 & 7.61 & 8.08 \\
\hline & 0.00 & 0.00 & 100.00 & 100.00 & 100.00 & 9.46 & 00.00 \\
\hline & & & & & & & 3.07 \\
\hline & & 9 & 5 & .20 & 29 & 5.88 & 9.53 \\
\hline & 2 & .87 & 9.20 & 9.11 & 9.19 & 94.41 & 99.24 \\
\hline & 30 & .44 & 79.32 & 77.35 & 8.65 & 5.57 & 82.58 \\
\hline & & & .93 & 12 & 9.01 & 9.54 & 8.90 \\
\hline & & & & & & 3.93 & 3.70 \\
\hline & & 32 & 76 & 6.19 & 84 & 82.06 & 8.91 \\
\hline & & & 50 & .62 & 7.90 & 8.83 & 8.45 \\
\hline & & 48 & 81 & 1.37 & 6.14 & 3.03 & 96.33 \\
\hline waveform- & & .46 & .50 & 80.85 & 86.98 & 90.77 & 89.87 \\
\hline zoo & 88.48 & 81.00 & 88.00 & 80.57 & 80.81 & 87.05 & 81.19 \\
\hline
\end{tabular}

\section{Conclusions and Future Work}

In this paper, we present a statistical technique, shrinkage, and an instancebased method, WPE, to improve the ranking performance of decision trees, which is measured by AUC. The class probability estimate with shrinkage is a linear interpolation for the local probability estimates on each node along the path from leaf to root. Algorithm DIW is proposed to determine the interpolating weights used in shrinkage. WPE produces the distinct probability estimates for the instances on the same node. In order to compensate for the deficiencies of shrinkage and WPE, we combine them together. In this process, we use WPE to generate distinct probability estimates on each node in training and testing processes, and also use shrinkage to return the final class probability estimate. The experiments show that decision tree algorithms with shrinkage and WPE outperform the original ones, and that the decision tree algorithms with this combination significantly outperform the original ones and other state-of-theart techniques proposed to enhance the ranking performance of decision trees.

In our future research, we will study other local probability estimation methods in decision trees. We notice that naive Bayes model also generates distinct probability estimates for different instances falling into the same node. In shrinkage, deploying naive Bayes model along a path to estimate class probabilities may produce good ranking performance. 


\section{References}

1. Bahl, L., Brown, P., deSouza, P., Mercer, R.: A tree-based statistical language model for natural language speech recognition. IEEE Transactions on Acoustics, Speech and Signal Processing. (1989) bf 37, 1001-1008

2. Bauer, E., Kohavi, R.: An empirical comparison of voting classification algorithms: Bagging, boosting and variants. Artificial Intelligence 36 (1989) 105-142

3. Bradley, A. P.: The use of the area under the ROC curve in the evaluation of machine learning algorithms. Pattern Recognition 30 (1997) 1145-1159

4. Buntine, W.: Learning classification trees. Artificial Intelligence frontiers in statistics. Chapman \& Hall, London (1993) 182-201

5. Ferri, C., Flach, P. A., Hernandez-Orallo, J.: Improving the AUC of Probabilistic Estimation Trees. Proceedings of the 14th European Conference on Machine Learning (ECML2003). Springer (2003)

6. Hand, D. J., Till, R. J.: A Simple Generalisation of the Area Under the ROC Curve for Multiple Class Classification Problems. Machine Learning 45 (2001) 171-186

7. Hastie, T., Pregibon, L.: Shrinking Trees. AT \& T Bell Laboratories (1990)

8. Ling, C. X., Huang, J., Zhang, H.: AUC: a statistically consistent and more discriminating measure than accuracy. Proceedings of 18th International Conference on Artificial Intelligence (IJCAI-2003). Morgan Kaufmann (2003) 329-341

9. Ling, C. X., Yan, R. J.: Decision tree with Better Ranking. Proceedings of the Twentieth International Conference on Machine Learning (ICML2003). AAAI Press (2003)

10. McCallum, A., Rosenfeld, R., Mitchell, T., Ng, A. Y.: Improving text classification by shrinkage in a hierarchy of classes. Proceedings of the 15th International Conference on Machine Learning. Morgan Kaufmann (1998) 359-367

11. Pazzani, M., Merz, C., Murphy, P., Ali, K., Hume, T., Bunk, C.: Reducing misclassification costs. Proceedings of the Eleventh International Conference on Machine Learning. Morgan Kaufmann (1994) 217-225

12. Provost, F., Domingos, P.: Tree Induction for Probability-based Ranking. Machine Learning. Kluwer Academic Publishers (2002)

13. Provost, F., Fawcett, T., Kohavi, R.: The case against accuracy estimation for comparing induction algorithms. Proceedings of the Fifteenth International Conference on Machine Learning. Morgan Kaufmann (1999) 445-453

14. Provost, F., Fawcett, T.: Analysis and visualization of classifier performance: Comparison under imprecise class and cost distributions. Proceedings of the Third International Conference on Knowledge Discovery and Data Mining (KDD-97). AAAI Press (1997) 43-48

15. Quinlan, J. R.: C4.5 Programs for Machine Learning. Morgan Kaufmann, San Mateo, CA (1993)

16. Witten, I. H., Frank, E.: Data mining-practical machine learning tools and techniques with java implementation. Morgan Kaufmann, San Mateo, CA (2000)

17. Zadrozny, B., Elkan, C.: Obtaining calibrated probability estimates from decision trees and Naive Bayesian classifiers. Proceedings of the 18th International Conference on Machine Learning. Morgan Kaufmann (2001) 609-616 